\title{
O Uso de Mobile Learning e Metodologias Ativas no Contexto Educacional
}

\section{Aline Verardo Corrêa ${ }^{4}$, Delfa Mercedes Huatuco Zuasnabar ${ }^{1,2}$, Miguel Raymundo Flores Santibanez ${ }^{1,2}$, Sandro José Ribeiro da Silva ${ }^{1,3}$, Lucas Plautz Prestes ${ }^{4}$, Patrícia Fernanda da Silva ${ }^{1}$}

${ }^{1}$ Programa de Pós Graduação em Informática na Educação - Universidade Federal Rio Grande do Sul

2 -Departamento de Ciência da Computação - Universidade Federal de Roraima

${ }^{3}$ Instituto Federal de Educação, Ciência e Tecnologia do Rio Grande do Sul

${ }^{4}$ Programa de Pós Graduação em Educação - Universidade Federal do Rio Grande do Sul

\begin{abstract}
This article aims to reflect on the use of mobile devices and the Active Methodologies approach, which allows students to position themselves in front of the classes as protagonists, building their own learning as an alternative of pedagogical innovation to the current reality. Therefore, a qualitative research was conducted, considering a descriptive, interpretative and deductive approach, allowing to understand the phenomena within their context and to discover the connections between the presented items. As the results point to use their use, they propose educational policies for the implementation of these technologies and methodological approaches to foster the creation of mobile educational content, such as preparing or teacher for the integration of mobile technologies for educational purposes.
\end{abstract}

Resumo. Este artigo objetiva refletir sobre o uso de dispositivos móveis e a abordagem das Metodologias Ativas, que possibilitam aos alunos posicionarem-se diante das aulas como protagonistas, construindo seu próprio aprendizado, como uma alternativa de inovação pedagógica para a realidade atual. Para tanto, foi realizada uma pesquisa qualitativa, considerando uma abordagem descritiva, interpretativa e dedutiva, possibilitando compreender os fenômenos dentro de seu contexto $e$ descobrindo ligações entre conceitos apresentados. Como resultados apontamos a regulamentação do seu uso, propondo políticas educacionais de implementação destas tecnologias e da abordagem metodológica, para fomentar a criação de conteúdos educacionais móveis, assim como, preparar o professor para a integração das tecnologias móveis para fins educacionais.

\section{Introdução}

A era da tecnologia digital transformou e vem transformando o modo de vida das pessoas, facilitando o acesso à informação e viabilizando a expansão da cultura digital. 
VIII Congresso Brasileiro de Informática na Educação (CBIE 2019)

Anais dos Workshops do VIII Congresso Brasileiro de Informática na Educação (WCBIE 2019)

A sociedade exige cada vez mais indivíduos pensantes, capazes de sentir, agir e reagir de forma ampla, profunda e rápida. Para tanto, é necessário engajamento e autonomia para lidar com suas próprias experiências e aplicá-las quando necessário no seu cotidiano.

Sobre o enfoque da era da tecnologia digital que vivenciamos e das transformações dela decorrentes, inovações pedagógicas são requeridas, porque as aulas expositivas não mais conseguem atrair e atingir as expectativas da sociedade contemporânea.

Chamar a atenção dos alunos e mantê-los envolvidos são pontos essenciais para o processo de aprendizagem e desenvolvimento do pensamento crítico e neste sentido, as Metodologias Ativas colocam os alunos no centro desse processo, tornando-os protagonistas da descoberta, em vez de apenas receptores passivos de informações, já o professor desempenha um papel de ativador da aprendizagem.

Em situações de ensino aprendizagem rumo às novas descobertas destacamos a integração das tecnologias digitais, usando os aplicativos móveis que superam as limitações de tempo e espaço e as metodologias de aprendizagem ativas, que possibilitam potencializar o ensino.

Diferentes estratégias de ensino oportunizam criar um ambiente de aprendizado ativo e envolver os alunos. As evidências atuais, indicam que o aprendizado ativo melhora a compreensão, a retenção de informações, sendo eficaz no desenvolvimento de habilidades cognitivas de ordem superior, no entanto, a adoção de abordagens com metodologias ativas ainda é baixa.

Com base na a teoria de aprendizagem construtivista, a aprendizagem ativa, que enfatiza o fato de que os aprendizes constroem seu próprio conhecimento, está relacionada com a aprendizagem experiencial a qual amplamente descreve alguma aprendizagem a partir de uma experiência direta. A ideia principal na aprendizagem é que aprendemos melhor quando podemos ver a utilidade do que aprendemos e conectá-lo ao mundo real.

Aprendizagem ativa é o conjunto de práticas pedagógicas que abordam o processo de aquisição de conhecimentos, habilidades, valores e atitudes por qualquer estratégia educacional que envolva os alunos no processo, levando-os a atividades e debates que os ajudem a pensar de forma crítica ou criativa, expressar idéias através da escrita, atitudes e valores, fornecer e receber feedbacks ou refletir sobre seu processo de aprendizagem. Este conceito inclui todas as práticas que ajudam os alunos a aumentar a retenção de informações, realizando pequenos exercícios escritos relacionados ao conteúdo abordado ou realizando exercícios em grupo complexos aplicados a situações da vida real e/ou a novos problemas. Portanto, a aprendizagem ativa abrange um amplo e variado conjunto de técnicas ou métodos que geralmente exigem que os alunos realizem atividades proativas significativas, estando cientes e responsáveis pelo que está sendo feito (PRINCE, 2004).

A tecnologia móvel voltada para a prática pedagógica promove uma aprendizagem criativa, crítica, personalizada, compartilhada, facilita a interação e estimula os alunos a uma aprendizagem significativa. A principal característica da 
VIII Congresso Brasileiro de Informática na Educação (CBIE 2019)

Anais dos Workshops do VIII Congresso Brasileiro de Informática na Educação (WCBIE 2019)

aprendizagem móvel é a ubiquidade, é dizer que permite o desenvolvimento do processo de ensino e aprendizagem em qualquer instante e lugar (KONOPKA, 2015).

Os autores do presente trabalho incentivam a reflexão sobre as práticas tradicionais de ensino e sugerem que as metodologias ativas possam ser uma opção para atender às necessidades atuais de ensino. Para tanto, apresenta alguns conceitos visando promover reflexões acerca das metodologias ativas e o uso de tecnologias digitais para suscitar a aprendizagem e construção de conhecimentos.

Este artigo está estruturado da seguinte forma: uma breve introdução, às Tecnologias Digitais e o uso de Mobile Learning, as Metodologias Ativas, à metodologia de trabalho, as aprendizagens baseadas em Metodologias Ativas e Tecnologias Digitais, as considerações finais e o referencial teórico que deu aporte ao referido trabalho.

\section{Tecnologias Digitais: 0 uso de Mobile Learning}

As relações com a tecnologia estão em constante movimento, hoje a mobilidade da comunicação é algo presente no cotidiano das pessoas. Dentre as tecnologias móveis o celular é o mais difundido, modificando a relação homem - máquina de tal forma, em que ocorrem entrelaçamentos até o ponto onde possa não existir uma separação clara.

Em um congresso a Professora Dra. Edméa chama atenção ao fato de que "Produzimos em movimento com os aparelhos celulares" (SANTOS, ENDIPE, 2018). Os computadores desktops, estão cada vez mais escassos nas residências das pessoas, as crianças da era digital já nascem podendo ter contato com celulares e grande parte de suas interações são através deles ou através de tablets.

Nesse cenário, a escola precisa pensar em "[...] promover formas de educação e letramento midiático que auxiliem as crianças a desenvolver as habilidades necessárias para se tornarem participantes plenos da sua cultura." (JENKINS, 2008, p. 331). Existe muito a ser feito e modificado, principalmente "Na questão do uso dos dispositivos móveis em sala de aula [onde] existe muita ignorância, sendo preciso utilizar como produção do conhecimento." (BLINKSTEIN, CBIE, 2018). E nesse sentido um estudo da UNESCO indica

\footnotetext{
"[...] Uma das barreiras mais resistentes ao desenvolvimento da aprendizagem móvel é a falta de educadores treinados que possam efetivamente incorporar o uso de tecnologias móveis na sala de aula. tanto os professores que já exercem a profissão quanto aqueles que estão entrando no mercado agora precisam de formação e treinamento para aprender a projetar intervenções inovadoras de aprendizagem móvel. Os formuladores de políticas precisam desenvolver estratégias para ajudar os professores a compreender melhor as complexas relações entre tecnologia móvel, pedagogia, projeção e implementação." (UNESCO, 2014, p. 51).
}

Esse estudo da UNESCO foi desenvolvido com o objetivo de uma construção de políticas para inserção das tecnologias móveis no processo educativo em 5 sub regiões 
do mundo ${ }^{1}$, e nos indica a necessidade atual de se quebrar barreiras existentes junto aos educadores em relação a utilização da tecnologia móvel, bem como da organização dos órgãos políticos.

O termo "treinados" da citação acima, pode levantar indagações, pois acredita-se que os professores precisam de formação e não apenas de um treino para saber incorporar as tecnologias ao contexto escolar. A formação tanto inicial, como a continuada precisa ser pensada no sentido de um professor e aluno que são reflexivos, e que podem ser desacomodados e desafiados a pensar na utilização de metodologias que realmente abram possibilidades de aprender.

Pensando em um professor que tenha desejo em modificar sua prática, e proporcionar mudanças no modo em que seus alunos constroem conhecimentos, com um perfil diferenciado, de docente que não se conforma com uma educação que visa reprodução, cópia e repetição pura e simples, que se abre a possibilidades de mudanças na educação. Mudanças estas principalmente em relação à utilização pedagógica da tecnologia, favorecendo a ultrapassagem de limites, onde o fazer fique ampliado ao atingir êxito, chegando a uma compreensão do processo de aprendizagem vivenciado. Este docente projetado para o futuro passa por uma desconstrução de modelos antigos e arcaicos de ensino, favorecendo para a construção de um professor para "Educar na era Digital" (BATES, 2017).

\section{Metodologias Ativas}

De acordo com Morán (2015), a educação está em um impasse diante de inúmeras mudanças na sociedade: é necessário evoluir e fazer com que todos aprendam de forma competente e construtiva. Dentro deste contexto, os estudantes têm a clareza do acesso a informação que as metodologias tradicionais não possuem suporte, assim não atingindo a demanda destes alunos quando relacionado ao seu processo de aprendizagem.

Desta forma, as atuais demandas sociais exigem muito mais do docente em sala de aula, não apenas uma nova postura, mas novas formas de transmitir o seu conhecimento e fazer com que os discentes a absorvam e entendam de forma que seja familiar ao seu cotidiano e com as condições sociais que os mesmos estão inseridos. (DIESEL, BALDEZ e MARTINS; 2017).

Como solução a esta adversidade no âmbito educacional, surgem os conceitos de Metodologias Ativas na sala de aula. Estas se conceituam no processo de transmissão de conhecimentos em que o aluno não é apenas o observador como na metodologia expositiva tradicional, mas assume um papel ativo na construção de sua educação, estimulando a busca por conhecimento de forma autônoma, concomitantemente promovendo processos interativos e de autoaprendizagem (LEITE, 2018; MOURÃO, 2017).

As metodologias ativas possuem inúmeras formas de utilização, tal como exemplo a abordagem da sala de aula invertida, que propõe que os alunos estudem as informações do conteúdo em suas casas, e na escola façam suas atividades complementares de debates com os colegas de classe, promovendo assim durante a aula

\footnotetext{
${ }^{1}$ Sub-regiões: África e Oriente Médio, Ásia, Europa, América Latina, América do Norte.
} 
um espaço para que o aluno possa tirar dúvidas sobre o conteúdo, que surgiram em casa, ao tentar fazer exercícios, reflexões ou as tarefas que foram propostas.

O tempo em sala de aula é otimizado, e o aluno tem à oportunidade questionar o professor sobre o que não entendeu. Esta abordagem metodológica proporciona que o aluno possa em casa elencar as suas dificuldades e em sala de aula, aproveitar o tempo com o professor para tentar saná-las.

Outro exemplo de abordagem de metodologia ativa em sala de aula que pode ser resumidamente citado é a Instrução por Pares, que consiste na leitura prévia de determinado conteúdo do currículo, sucedida por debates e perguntas conceituadas no que foi estudado, feitas pelo docente. Esta estratégia pedagógica promove uma reflexão e pensamento crítico por parte do estudante, assim como, uma maior interação com o restante da turma juntamente com a troca de conhecimentos observados distintamente.

\section{Metodologia}

Este artigo começa com a apresentação de uma visão geral dos conceitos de tecnologias digitais: o uso de mobile learning e à abordagem de metodologias ativas, que possuem como base textos, artigos e trabalhos científicos.

Para dar continuidade, segue um discurso sobre a aprendizagem baseada no uso de mobile learning e metodologias ativas.

Algumas Questões-chaves que abordam a relação entre mobile learning e metodologias ativas foram identificadas por meio de revisões da literatura, onde as mesmas são utilizadas no contexto educacional.

A metodologia que norteia esta pesquisa é qualitativa, considerando uma abordagem descritiva, interpretativa e dedutiva, que pretende provocar o leitor e levá-lo à reflexão dos conceitos, temas e questões abordados no texto, visando mostrar a importância e formas de utilização no contexto educacional.

Visando que o leitor também possa refletir, pensar e posicionar-se criticamente diante da utilização das tecnologias digitais e das metodologias ativas, algumas questões norteadoras foram levantadas, e espera-se que ao final desta pesquisa, estas questões possam ser de certa forma, respondidas. São elas:

Questão 1. De que maneira o uso das tecnologias móveis podem contribuir no processo de aprendizagem ativa dos alunos?

Questão 2. Os professores estão preparados para planejar e desenvolver suas aulas usando a tecnologia móvel e as metodologias ativas?

Questão 3. Porque tratar de incorporar dispositivos móveis como ferramenta educativa?

\section{Aprendizagem baseada no uso de Mobile Learning e Metodologias Ativas}

O uso de metodologias ativas está apoiado no princípio da autonomia e em sala de aula, sendo de vital importância devido ao impacto que podem ocasionar à aprendizagem dos alunos. Portanto, uma abordagem criteriosa exige que os professores se tornem conhecedores das maneiras pelas quais as estratégias de Metodologias Ativas têm sido utilizadas com sucesso em muitas áreas. 
VIII Congresso Brasileiro de Informática na Educação (CBIE 2019)

Anais dos Workshops do VIII Congresso Brasileiro de Informática na Educação (WCBIE 2019)

Para Dos Santos \& Ferrari (2017), as seguintes atividades de Metodologias Ativas podem ser utilizadas no contexto educacional:

- Discutir temas e tópicos de interesse para a formação profissional;

- Geração de ideias (brainstorming) para a resolução de problemas;

- Estudo de casos relacionados com áreas de formação profissional específica;

- Trabalho em equipe com tarefas colaborativas;

- Debater temas da atualidade e da comunidade em que se está inserido;

- Produzir mapas mentais ou conceituais para esclarecer e aprofundar conceitos e ideias;

- Realizar modelagem e simulação de processos e sistemas típicos da área de formação;

- Elaborar questões de pesquisa na área científica e tecnológica;

- Criar sites ou redes sociais visando aprendizagem colaborativa.

As ferramentas para aprender nas instituições do século XXI precisam refletir sobre as expectativas em mudança de como, quando e onde aprendemos, e que eles devem motivar os alunos a se tornarem mais ativos e envolvidos em seu aprendizado.

Segundo Baharon (2013), a aprendizagem móvel apresenta algumas características que podem potencializar a sua utilização no contexto educacional, tais como:

- Sensível ao contexto, capacidade de envolver a aprendizagem no contexto do aluno; localizado, situado, autêntico;

- Conveniência, imediatismo, na hora certa, velocidade de acesso à informação, capacidade instantânea;

- Portátil, permite aprender a qualquer hora e em qualquer lugar;

- Flexível;

- Conveniente de usar durante momentos ociosos, fácil de transportar, disponibilidade conveniente de informações;

- Amigável ao usuário, simples de aprender e usar, familiar;

- Colaborativo, constrói comunidades de aprendizagem;

- Individualístico, centrado no usuário;

- Abrangente no uso do dispositivo móvel;

- Pessoal, privado;

- Aumenta a motivação e o engajamento, interatividade, capacita os alunos a participar;

- Facilita a comunicação, interatividade social, aumenta a interação, senso de conectividade; 
VIII Congresso Brasileiro de Informática na Educação (CBIE 2019)

Anais dos Workshops do VIII Congresso Brasileiro de Informática na Educação (WCBIE 2019)

- Acessibilidade do dispositivo.

De acordo com Litchfield (2007), o smartphone pode oferecer diferentes tipos de atividades de aprendizado através do uso de vários aplicativos móveis. $\mathrm{O}$ aprendizado móvel pode oportunizar experiências únicas de aprendizado que seriam diferentes de outras formas de tecnologias educacionais. Para o autor, a aprendizagem móvel, possibilita que ao aluno diversas atividades, dentre elas destacam-se:

- Acesse os materiais do curso e receba/entregue notificações de alertas e lembretes;

- Comunicação imediata e criação da comunidade (colaboração);

- Acesse ou crie pequenas notas em multimídia (texto, visual, áudio);

- Coletar dados ou capturar informações, fotos ou vídeos em contexto;

- Colaborar para compartilhar e discutir em contexto;

- Capture fotos ou crie um blog de fotos em contexto.

O aprendizado móvel trata sobre uma mudança na abordagem filosófica do professor para o professor, e não é simplesmente aplicar os requisitos de projeto de aprendizagem ao ambiente de aprendizado móvel. Isso significa que as iniciativas de aprendizagem móvel devem estabelecer seus próprios requisitos de projeto para suportar suas características (DEROUNIAN, 2017).

Ao mesmo tempo, o autor salienta que alguns requisitos para uso das tecnologias são necessários por parte do professor e devem ser observados antes e durante as atividades que visam utilizar as tecnologias digitais. Para tanto, por meio de uma revisão de um guia de aprendizagem móvel, alguns aspectos importantes são levantados, visando que o professor possa estar atento e busque desenvolver as atividades de acordo com estas orientações:

- Disponibilidade de tecnologia;

- Avaliação da disposição dos alunos de usar seus próprios dispositivos móveis em uma variedade de atividades de aprendizagem;

- Conectividade através de redes móveis e acesso à rede sem fio;

- Personalizar empregando o próprio dispositivo móvel dos alunos;

- Suporte institucional, como recursos, treinamento de pessoal e disponibilidade e manutenção de hardware;

- Conhecimento das limitações e benefícios dos dispositivos móveis;

- Foco nas características da aprendizagem móvel;

- Entender o ambiente de aprendizagem em que o design ocorre; contextos móveis

- Aprender sobre o contexto;

- Integrar-se ao currículo, às experiências dos alunos e à própria iniciativa de aprendizado móvel; 
VIII Congresso Brasileiro de Informática na Educação (CBIE 2019)

Anais dos Workshops do VIII Congresso Brasileiro de Informática na Educação (WCBIE 2019)

- Uma análise crítica e cuidadosa sobre como os aplicativos de aprendizado móvel disponíveis podem ser usados para atender a objetivos específicos de aprendizado é essencial para garantir o sucesso de qualquer implementação de aprendizado móvel;

- Escolha apropriada de dispositivos móveis e software;

- Investigar protocolos técnicos para fazer o download de dispositivos móveis para computadores.

\section{Conclusão}

Conforme considerações da UNESCO (2014), às tecnologias móveis, que num futuro próximo, estarão integradas à educação a tal ponto que até o termo aprendizagem móvel ou mobile learning caia em desuso devido à compreensão dos conceitos de aprendizagem de forma holística.

Diante disso, é preciso buscar preparo para utilizar diferentes metodologias ativas, que proporcionem a aprendizagem e à construção de conhecimentos de forma autônoma pelos alunos e ainda apoiadas no uso de tecnologias digitais.

É imprescindível que o professor conheça, e possa aceitar as tecnologias móveis no contexto educacional, regulamentando seu uso, propondo políticas educacionais de implementação destas tecnologias, difundindo exemplos de uso de aprendizagem móvel, fomentando a criação de conteúdos educacionais móveis, assim como, preparando o aluno para utilizar as tecnologias digitais com fins educacionais.

Neste ambiente de aprendizagem ativa, o professor atua como orientador, supervisor, facilitador do processo de aprendizagem, e não apenas como fonte única de informação e conhecimento. Dessa maneira, o aprendiz precisa ser um participante ativo para que ocorra a aprendizagem.

As metodologias ativas podem contribuir muito para a aprendizagem e para a construção de conhecimentos por parte do aluno, desenvolvendo possibilidades de trabalhos colaborativos, reflexão, desenvolvimento da criticidade, busca e autonomia por parte dos alunos.

Em contrapartida, o professor ao aliar o uso das tecnologias digitais e dispositivos móveis, possibilita ao aluno conectar-se e buscar por diferentes conhecimentos, aprender em qualquer tempo e lugar, independente de espaço físico e distância, abordar temas atuais e ainda aprender em um contexto e com o uso de uma ferramenta com a qual já tem grande familiaridade, o seu dispositivo móvel.

Para dar continuidade à pesquisa, pretende-se como trabalho futuro buscar e testar ferramentas que possam ser aliadas no uso das tecnologias digitais e no desenvolvimento das metodologias ativas, suscitando nos alunos a aprendizagem.

\section{Referências}

Baharon, S. S. (2013) Designing Mobile Learning Activities in the Malasian HE in the Context: a social constructivist approach. Stanford Business School, University of Salford, Salford, UK. 
VIII Congresso Brasileiro de Informática na Educação (CBIE 2019)

Anais dos Workshops do VIII Congresso Brasileiro de Informática na Educação (WCBIE 2019)

Bates, A. W. Tony. (2017), Educar na era digital [livro eletrônico]: design, ensino e aprendizagem; [tradução João Mattar].1. ed.São Paulo: Artesanato Educacional.

Blinkstein, Paulo. (2018). Palestra Menos Vale do Silício, mais Papert e Paulo Freire: futuros possíveis para a tecnologia educacional no Brasil. CBIE - Congresso Brasileiro de Informática na Educação,Fortaleza.

Boulic, R. and Renault, O. (1991) “3D Hierarchies for Animation”, In: New Trends in Animation and Visualization, Edited by Nadia Magnenat-Thalmann and Daniel Thalmann, John Wiley \& Sons ltd., England.

Derounian, J. G. (2017) Mobiles in class. Active Learning in Higher Education, 1-12

Dyer, S., Martin, J. and Zulauf, J. (1995) "Motion Capture White Paper", http://reality.sgi.com/employees/jam_sb/mocap/MoCapWP_v2.0.html, December.

Dos Santos, C. M. R. and Ferrari, M. A. (2017). Aprendizagem ativa: Contextos e experiências em comunicação, Universidade Estadual Paulista, Faculdade de Arquitetura, Artes e Comunicação, Bauru.

Holton, M. and Alexander, S. (1995) "Soft Cellular Modeling: A Technique for the Simulation of Non-rigid Materials", Computer Graphics: Developments in Virtual Environments, R. A. Earnshaw and J. A. Vince, England, Academic Press Ltd., p. $449-460$.

Jenkins, Henry (2018). Cultura da Convergência. São Paulo: Aleph.

Konopka, C. L., Adaime, M. A., Mosele, P. H. (2015). Active Teaching and Learning Methodologies: Some Considerations. Creative Education, 6, 1536-1545 Published Online August 2015 in SciRes. http://www.scirp.org/journal/ce http://dx.doi.org/10.4236/ce.2015.614154.

Litchfield, A., Dyso, L. E and Lawrence, E. (2007). Directions for m-learning research to enhance active learning. Proceedings ascilite Singapore.

Prince, M. J. (2004). Does Active Learning Work? A Review of the Research. Journal $\begin{array}{llll}\text { of Engineering } & \text { Education, } 231 .\end{array}$ http://dx.doi.org/10.1002/j.2168-9830.2004.tb00809.x

Unesco. (2014). O futuro da aprendizagem móvel: implicações para planejadores e gestores de políticas. Brasília, 2014. 